\title{
Wavelength-Tunable Laser for Signal Remodulation in WDM Access Networks Using DPSK Downlink and OOK Uplink
}

\author{
C. H. Yeh, C. W. Chow, Member, IEEE, F. Y. Shih, Student Member, IEEE, C. H. Wang, Student Member, IEEE, \\ Y. F. Wu, and S. Chi
}

\begin{abstract}
In this investigation, a simple wavelength-tunable laser based on a Fabry-Pérot laser diode (FP-LD) and an erbium-doped fiber amplifier to serve as a downlink signal in a colorless wavelength-division-multiplexed passive optical network (PON) is proposed and experimentally demonstrated. The tuning range of the proposed laser is between 1529.48 and $1560.72 \mathrm{~nm}$, and the output performance of proposed laser is discussed. Colorless operation is implemented by using an FP-LD and a reflective semiconductor optical amplifier in each optical network unit for uplink signal remodulation, respectively. In addition, error-free data signal remodulation using $10-\mathrm{Gb} / \mathrm{s}$ downlink differential phase-shift keying and 2.5-Gb/s uplink on-off keying is achieved in a $25-\mathrm{km}$ reach $P O N$.
\end{abstract}

Index Terms-Fabry-Pérot laser diode (FP-LD), millimeterwave, radio-over-fiber.

\section{INTRODUCTION}

$\mathbf{T}$ ODAY, fiber-to-the-x (FTTx) is a promising technology for the last mile optical access networks due to the great capacity, high flexibility and cost-effectiveness [1]. The rapid growth of triple-play services (internet data, voice, and video) is demanding more and more bandwidth. The wavelength-division-multiplexed passive optical network (WDM-PON) is one of the potential and attractive solutions for the next-generation FTTx due to its high capacity and cost-effectiveness [2]-[5]. However, the cost of WDM-PON is relatively high due to the need of a wavelength-specific laser source in each optical network unit (ONU). In addition, tunable laser sources are required in the dynamic wavelength assignment WDM networks [5]-[9]. As a result, tunable lasers still need to be further developed for WDM-PONs. For the "colorless" operation in the WDM-PON, several optical-injection methods with signal remodulation for uplink transmitters

Manuscript received March 10, 2009; revised July 23, 2009. First published September 25, 2009; current version published October 30, 2009. The work of C. W. Chow was supported by the National Science Council, Taiwan, R.O.C., under Contract NSC 96-2218-E-009-025-MY2, 97-2221-E-009-038-MY3.

C. H. Yeh is with the Information and Communications Research Laboratories, Industrial Technology Research Institute, Chutung, Hsinchu 31040, Taiwan (e-mail: depew@itri.org.tw).

C. W. Chow, F. Y. Shih, C. H. Wang, and Y. F. Wu are with Department of Photonics and Institute of Electro-Optical Engineering, National Chiao Tung University, Hsinchu 30010, Taiwan.

S. Chi is with Department of Photonics Engineering, Yuan Ze University, Chungli, Taoyuan 32003, Taiwan.

Color versions of one or more of the figures in this letter are available online at http://ieeexplore.ieee.org.

Digital Object Identifier 10.1109/LPT.2009.2032072 have been reported and studied, such as using spectrum-sliced light-emitting diodes [7], light-injected reflective semiconductor optical amplifiers (RSOAs) [3], and injection-locked Fabry-Pérot laser diodes (FP-LDs) [4], [5]. Here, the signal remodulation by reusing the downlink wavelength for generating the uplink signal can also reduce the cost [3]-[5].

In this study, we propose a simple wavelength-tunable laser based on an FP-LD and an erbium-doped fiber amplifier (EDFA) in the optical line terminal (OLT) acting as a downlink signal source. The downlink wavelength is modulated at $10-\mathrm{Gb} / \mathrm{s}$ differential phase-shift keying (DPSK) format and the uplink signal uses $2.5-\mathrm{Gb} / \mathrm{s}$ on-off keying (OOK) remodulation by wavelength-injected FP-LD and wavelength-injected RSOA in each ONU, respectively. Compared with [5], our proposed tunable laser is not only simple, but also can achieve a $2.5-\mathrm{Gb} / \mathrm{s}$ uplink remodulation rate under $25-\mathrm{km}$ single-mode-fiber transmission. Compared with our previously proposed laser source [10], the proposed laser in this letter can produce larger output power, and have more stable and easily adjustable single-wavelength output. In the past study [10], in order to achieve a stable output, we needed to carefully control the temperature and polarization state of the FP-LD due to self-injected operation is used. Here, the network performance of the experiment is also analyzed and discussed.

\section{EXPERIMENT AND DISCUSSION}

Fig. 1 illustrates the proposed colorless WDM-PON architecture using an FP-LD-based wavelength-tunable laser for the $10-\mathrm{Gb} / \mathrm{s}$ downlink signal. The proposed laser consists of a multilongitudinal-mode (MLM) FP-LD, a tunable bandpass filter (TBF), a polarization controller (PC), and an EDFA module. Here, the EDFA is constructed by a $980-\mathrm{nm}$ pump laser with $70 \mathrm{~mW}$, a 10-m EDF, a 980/1550-nm WDM coupler (WC), and an optical isolator (OIS). This EDFA module has a saturation power of $\sim 18 \mathrm{dBm}$ and noise figure of $6 \mathrm{~dB}$. The 3-dB bandwidth and tuning range of TBF are 0.4 and $35 \mathrm{~nm}(1525-1560 \mathrm{~nm})$, respectively. The PC is employed to control the output polarization state of the proposed laser. In the experiment, the bias current of the MLM FP-LD is $28 \mathrm{~mA}$ at a temperature of $24{ }^{\circ} \mathrm{C}$. An optical spectrum analyzer (OSA) with a $0.02-\mathrm{nm}$ resolution and a power meter are used to measure the output wavelength and output power, respectively. Fig. 2(a) shows the output spectrum of the MLM FP-LD with 1.1-nm mode spacing (observed at location "a") and the central wavelength (maximum peak power) is observed 


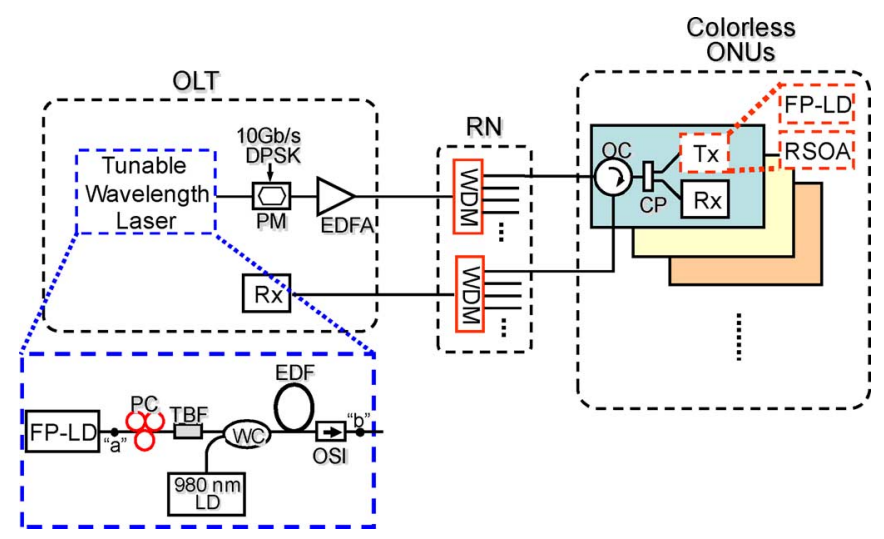

Fig. 1. Proposed wavelength-tunable laser for wavelength remodulation WDM-PON using DPSK downlink and OOK uplink.

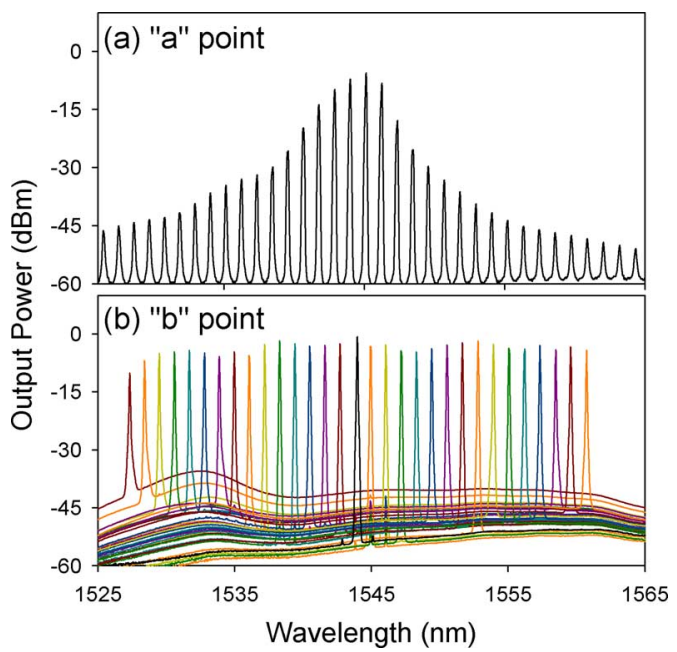

Fig. 2. (a) Originally output spectrum of MLM FP-LD used operates at $28 \mathrm{~mA}$ in the temperature of $24^{\circ} \mathrm{C}$. (b) Output spectra of proposed laser in the wavelengths of $1529.48-1560.72 \mathrm{~nm}$ with tuning step of $1.1 \mathrm{~nm}$.

at $1545.12 \mathrm{~nm}$. The TBF is used to align the corresponding mode of the MLM FP-LD, and the filtered backward amplified spontaneous emission (ASE) power of the EDFA module could be injected into the FP-LD. Then, the target mode of the FP-LD will be amplified after passing through the EDFA module. The injected ASE power at the input of the FP-LD was $-7 \mathrm{dBm}$. This generates a single-longitudinal-mode (SLM) output. The phase modulator (PM) encodes the $10-\mathrm{Gb} / \mathrm{s}$ DPSK signal. Another EDFA (saturation power of $\sim 23 \mathrm{dBm}$ and noise figure of $5 \mathrm{~dB}$ ) after the PM is used to compensate the transmission loss, as shown in Fig. 1. Fig. 2(b) presents the output spectra of proposed laser observing at location "b" in Fig. 1 in wavelength range from 1529.48 to $1560.72 \mathrm{~nm}$ with a tuning step of $1.1 \mathrm{~nm}$.

Fig. 3(a) shows the output power and the sidemode suppression ratio (SMSR) in the wavelength range from 1529.48 to $1560.72 \mathrm{~nm}$ with a $1.1-\mathrm{nm}$ tuning step. The output powers and SMSRs are between -1.8 and $0.3 \mathrm{dBm}$ and 37.2 and $49.7 \mathrm{~dB}$, respectively, in the effectively operating range, as shown in Fig. 3(a). The maximum output power is $0.3 \mathrm{dBm}$ with 46.4-dB SMSR at the wavelength of $1544.92 \mathrm{~nm}$. In Fig. 3(a), the minimum outputs are observed in both sides of the wavelength range. Since high SMSR can be achieved, we



Fig. 3. (a) Output power and SMSR spectra in the wavelength range of 1529. $48-1560.72 \mathrm{~nm}$ with a 1.1-nm tuning step. (b) Output variations of central wavelength and power at $1542.68 \mathrm{~nm}$ initially under 30 min observing time.

believe that the partition noise is highly suppressed. This can also be observed in the previous report of ASE injection locking [11]. The temperature of FP-LD used in the proposed laser is maintained at $24{ }^{\circ} \mathrm{C}$ in order to obtain the stable outputs. To demonstrate and analyze the performance of output power and wavelength stability, a short-term output stability test of the proposed laser is performed. The lasing single-mode wavelength is $1542.68 \mathrm{~nm}$ initially with $0.2-\mathrm{dBm}$ output power and the observation time is over $30 \mathrm{~min}$. The maximum variation of the central wavelength and output power in the proposed tuning laser are 0 and $0.9 \mathrm{~dB}$, respectively, as shown in Fig. 3(b). Moreover, the stabilized output of the proposed wavelength tunable laser is still maintained during $4 \mathrm{~h}$ of observation.

In addition, the temperature applied to the FP-LD could cause the output wavelength of the FP-LD to shift slightly. According to the measurement result, when the temperature variation $\Delta T= \pm 4^{\circ} \mathrm{C}$ of FL-LD, the lasing wavelength could be tuned in the $0.16-\mathrm{nm}$ range. Thus, the proposed laser could be continuously tunable by controlling the temperature of the FP-LD.

In the experiment, the output wavelength of the proposed laser is modulated at $10-\mathrm{Gb} / \mathrm{s}$ DPSK format via a $\mathrm{LiNbO}_{3} \mathrm{PM}$ for downlink traffic in the colorless WDM-PON. The PM is driven by a differentially precoded $10 \mathrm{~Gb} / \mathrm{s}$ at pseudorandom binary sequence (PRBS) nonreturn-to-zero (NRZ) with $2^{31}-1$ pattern length. The downlink DPSK signal launches into the ONU via an optical circulator (OC), and $10 \%$ of the downlink power is received by an optically preamplified receiver (Rx), consisting of a variable optical attenuator (VOA), an EDFA, a delayed interferometer (DI) for DPSK demodulation, and a 10-GHz PIN. Here, we use the FP-LD and RSOA in each ONU for uplink remodulation. The FP-LD and RSOA are wavelengthinjected by the residual power (90\%) of the downlink signal, and they are directly modulation at $2.5 \mathrm{~Gb} / \mathrm{s}$ by NRZ PRBS format with $2^{31}-1$ word length, respectively. In each ONU, the FP-LD-based transmitter has the same characteristic as the 


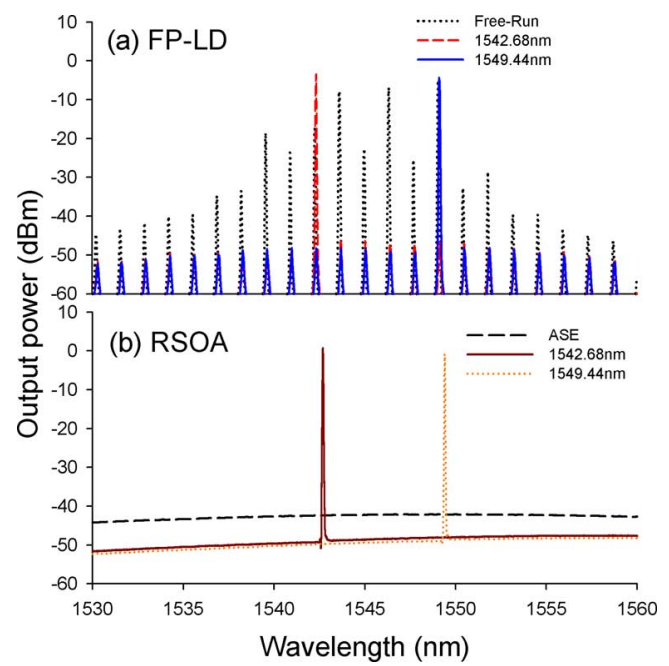

Fig. 4. Output spectra of (a) FP-LD and (b) RSOA without and with downlink injection under the two wavelengths of 1542.68 and $1549.44 \mathrm{~nm}$, respectively.

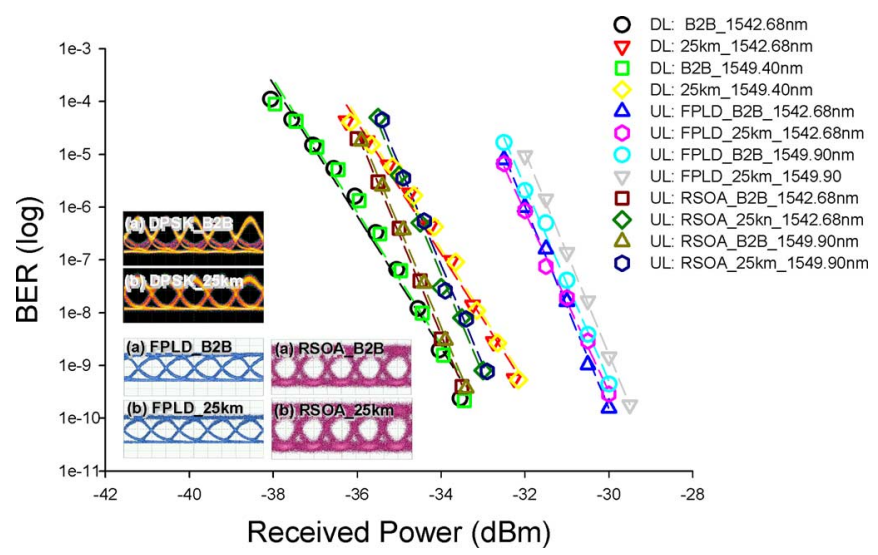

Fig. 5. BER measurements of the downstream and upstream signals at back-toback (B2B) and after 25-km fiber transmission without dispersion compensation. Inserts are the corresponding eye diagrams. DL: downlink.

FP-LD used in OLT. The operating currents of the FP-LD and the RSOA used are 26 and $70 \mathrm{~mA}$ at a temperature of $25^{\circ} \mathrm{C}$, respectively. Thus, Fig. 4(a) and (b) presents the output spectra of the FP-LD and the RSOA without and with the downlink injection at the wavelengths of 1542.68 and $1549.44 \mathrm{~nm}$, respectively. And the black dashed lines of Fig. 4 are the free-run output spectra of FP-LD and RSOA without any optical injection. In addition, in order to achieve a $2.5-\mathrm{Gb} / \mathrm{s}$ OOK uplink traffic by optical injection, the launched optical power into the FP-LD and RSOA should be larger than -16 and $-10 \mathrm{dBm}$, respectively.

To measure the bit-error-rate (BER) performance of the proposed PON architecture, we arbitrary select two wavelengths, 1542.68 and $1549.90 \mathrm{~nm}$, from the proposed wavelength-tunable laser source in the OLT for downlink traffic and signal remodulation. Fig. 5 presents the BER measurements of the downlink and uplink signals at back-to-back and after $25-\mathrm{km}$ fiber transmission without dispersion compensation. The insets of Fig. 5 are the corresponding eye diagrams at $1542.68 \mathrm{~nm}$. As shown in Fig. 5, error-free operation is observed in each case with clear and wide open eye. About 1.4-dB power penalty at a BER of $10^{-9}$ in the downlink DPSK signal is observed. In addition, we also obtain negligible power penalty in the uplink OOK signal after the $25-\mathrm{km}$ transmission using FP-LD and RSOA, respectively.

\section{CONCLUSION}

We proposed and experimentally demonstrated a simple wavelength-tunable laser based on an FP-LD and an EDFA to serve as a downlink signal in colorless WDM-PON. The output powers and SMSRs of the proposed laser are between -1.8 and $0.3 \mathrm{dBm}$, and 37.2 and $49.7 \mathrm{~dB}$, respectively, in the effective operating range from 1529.48 to $1560.72 \mathrm{~nm}$. Here, the colorless operation is implemented by using the FP-LD and RSOA in each ONU for uplink signal remodulation. Error-free remodulation using 10-Gb/s DPSK downlink and 2.5-Gb/s uplink OOK remodulation are achieved in a $25-\mathrm{km}$ reach WDM-PON without dispersion compensation. Since colorless reflective modulators are used, this can also reduce the cost of wavelength referencing and stabilization at the ONU. In addition, cost can be further reduced in the signal remodulation network by wavelength reuse. We believe the cost-effective network architecture can justify the cost of the ONU and OLT.

\section{REFERENCES}

[1] K. McCammon and S. W. Wong, "Experimental validation of an access evolution strategy: Smooth FTTP service migration path," in Proc. OFC, Anaheim, CA, 2007, Paper NThB3.

[2] Z. Xu, Y. J. Wen, W. D. Zhong, C. J. Chae, X. F. Cheng, Y. Wang, C. $\mathrm{Lu}$, and J. Shakar, "High-speed WDM-PON using CW injection locked Fabry-Perot laser diodes," Opt. Express, vol. 15, pp. 2953-2962, 2007.

[3] P. Healey, P. Townsend, C. Ford, L. Johnston, P. Townley, I. Lealman, L. Rivers, S. Perrin, and R. Moore, "Spectral slicing WDM-PON using wavelength-seeded reflective SOAs," Electron. Lett., vol. 37, pp. 1181-1182, 2001.

[4] W. Hung, C. K. Chan, L. K. Chen, and F. Tong, "An optical network unit for WDM access networks with downstream DPSK and upstream remodulated OOK data using injection-locked FP laser," IEEE Photon. Technol. Lett., vol. 15, no. 10, pp. 1476-1478, Oct. 2003.

[5] C. L. Tseng, C. K. Liu, J. J. Jou, W. Y. Lin, C. W. Shih, S. C. Lin, S. L. Lee, and G. Keiser, "Bidirectional transmission using tunable fiber lasers and injection-locked Fabry-Pérot laser diodes for WDM access networks," IEEE Photon. Technol. Lett., vol. 20, no. 10, pp. 794-796, May 15, 2008.

[6] F. T. An, K. S. Kim, D. Gutierrez, S. Yam, E. S. T. Hu, K. Shrikhande, and L. G. Kazovsky, "SUCCESS: A next-generation hybrid WDM/TDM optical access network architecture," J. Lightw. Technol., vol. 22, no. 11, pp. 2557-2569, Nov. 2004.

[7] D. K. Jung, H. Kim, K. H. Han, and Y. C. Chung, "Spectrum-sliced bidirectional passive optical network for simultaneous transmission of WDM and digital broadcast video signals," Electron. Lett., vol. 37, pp. 308-309, 2001.

[8] C. H. Yeh, C. W. Chow, C. H. Wang, F. Y. Shih, H. C. Chien, and S. Chi, "A self-protected colorless WDM-PON with $2.5 \mathrm{~Gb} / \mathrm{s}$ upstream signal based on RSOA," Opt. Express, vol. 16, pp. 12296-12301, 2008.

[9] C. H. Yeh, C. W. Chow, C. H. Wang, F. Y. Shih, Y. F. Wu, and S. Chi, "Using four wavelength-multiplexed self-seeding Fabry-Perot lasers for $10 \mathrm{Gbps}$ upstream traffic in TDM-PON," Opt. Express, vol. 16, pp. 18857-18862, 2008

[10] C. H. Yeh, F. Y. Shih, C. H. Wang, C. W. Chow, and S. Chi, "Costeffective wavelength-tunable fiber laser using self-seeding Fabry-Perot laser diode," Opt. Express, vol. 16, pp. 435-439, 2008.

[11] M. Fujiwara, H. Suzuki, K. Iwatsuki, and M. Sugo, "Noise characteristics of signal reflected from ASE-injected FP-LD in loopback access networks," Electron. Lett., vol. 42, pp. 111-112, 2006. 